\title{
Germanica
}

\section{Hybride Identitäten zwischen Wortlandschaften. Marica Bodrožićs Prosaband Sterne erben, Sterne färben}

Hybrid identities in the landscapes of words, the prose tale Sterne erben

Sterne färben (Stars inherit, stars fade) by Marica Bodrožić

Identités hybrides entre les paysages de mots. Le récit en prose Sterne erben

Sterne färben de Marica Bodrožić

\section{Raluca Rădulescu}

\section{(2) OpenEdition}

Journals

Édition électronique

URL : http://journals.openedition.org/germanica/1986

DOI : 10.4000/germanica. 1986

ISSN : 2107-0784

Éditeur

Université de Lille

Édition imprimée

Date de publication : 31 décembre 2012

Pagination : 63-74

ISBN : 9782913857308

ISSN : 0984-2632

\section{Référence électronique}

Raluca Rădulescu, « Hybride Identitäten zwischen Wortlandschaften. Marica Bodrožićs Prosaband

Sterne erben, Sterne färben », Germanica [Online], 51 | 2012, Online erschienen am: 14 Januar 2013, abgerufen am 06 Oktober 2020. URL : http://journals.openedition.org/germanica/1986 ; DOI : https:// doi.org/10.4000/germanica.1986

Ce document a été généré automatiquement le 6 octobre 2020.

(c) Tous droits réservés 


\title{
Hybride Identitäten zwischen Wortlandschaften. Marica Bodrožićs Prosaband Sterne erben, Sterne färben
}

\author{
Hybrid identities in the landscapes of words, the prose tale Sterne erben \\ Sterne färben (Stars inherit, stars fade) by Marica Bodrožić \\ Identités hybrides entre les paysages de mots. Le récit en prose Sterne erben \\ Sterne färben de Marica Bodrožić
}

Raluca Rădulescu

1 Marica Bodrožić wurde 1973 in Dalmatien geboren und hat Ihre Kindheit bei ihrem Großvater in einem dalmatinischen Dorf verbracht, während ihre Eltern schon in Deutschland lebten. 1983, drei Jahre nach dem Tode Titos, ist auch sie, als Zehnjährige, den Eltern nach Deutschland gefolgt. Sie studierte Kulturanthropologie und Slawistik in Frankfurt am Main, brach bald darauf das Studium ab, um sich ausschließlich dem Schreiben zu widmen. Ihre ersten literarischen Arbeiten wurden in der „Frankfurter Allgemeinen Zeitung“ und der Zeitschrift „manuskripte“ veröffentlicht. Im Sommer 2001 erhielt Marica Bodrožic das „Herman Lenz“-Stipendium. 2002 folgte dann der „Heimito von Doderer“-Förderpreis. Im selben Jahr veröffentlichte sie ihren ersten Erzählband Tito ist tot. 2003 wurden ihr der „Robert Bosch Stiftung“ Förderpreis sowie der "Adalbert von Chamisso" Förderpreis, 2006 das Jahresstipendium des Deutschen Literaturfonds und 2007 der Literaturpreis der Berliner Akademie der Künste überreicht. Zu ihrem Werk gehören noch die Romane Der Spieler der inneren Stunde (2005), Das Gedächtnis der Libellen (2010), der Erzählband Der Windsammler (2007), der Prosaband Sterne erben, Sterne färben (2007) und die Gedichtbände Ein Kolibri kam unverwandelt (2007), und Lichtorgeln (2008). Marica Bodrožić lebt heute als freie Autorin in Berlin.

Marica Bodrožićs 2007 erschienener Prosaband Sterne erben, Sterne färben ist ein Versuch, durch eine Hin und Her - Reise, die sowohl zurück in die Vergangenheit als auch weiter bis in die Gegenwart führt, eine seelische Biographie zu erkunden. In 
Anlehnung an Sartre lässt die Tiefenstruktur des Textes den Spracherwerb, den Umgang mit Sprache, das Hineinwachsen durch sie in eine Kultur, das über sich Hinauswachsen, „bis hin zum höchsten aller Sprachziele - Schriftstellerin werden“ ${ }^{\text {“ }}$ erkennen.

Dieser empfindungsbeladenen Topographie liegt die Frage nach dem Vorrang einer sprachlich erzeugten Seelenlandschaft zugrunde, in der Wörter verschiedener Sprachen und Dialekte zusammenwohnen, wobei jede nationale Prägung zugleich einen ins Emotionale gesteigerten Einfluss ausübt. Die Erzählerin stellt sich als Fragende und Suchende dar, die zwischen zwei Identitäten hin-und herwechselt. Jugoslawische Vergangenheit, die Ankunft im deutschen Aufnahmeland, das neue hessische Dorf, neuere Erfahrungen des Erwachsenen in europäischen Großstädten, alles kreist um das identitätserforschende Unterfangen, Trennlinien zu entdecken und zugleich Brücken zwischen Menschen und Kulturen zu schlagen.

Obwohl das reflektierende Ich von der These ausgeht, dass „erst in der deutschen Sprache“ das eigene Zuhause „selbst hörbar“ wird², was auf eine eindeutige Verortung der Identität verweist, lässt sich im Laufe des Buches näher feststellen, worauf diese eigentlich fußt. Die Verfasserin entwirft den Text so, als führte sie ein Tagebuch, in dem sie dem Leser Geständnisse macht, sie möchte ihn an ihren intimsten Erfahrungen teilhaben lassen. Dadurch sei ein kathartischer Prozess in die Wege geleitet, durch Schreiben erzielt sie die „Befreiung aus der Umzäunung der Biographie“" Strenge einer fremd zugesprochenen Lage, eines aufgezwungenen Status. In der Tat erweist sich aber die kritische Aneinanderreihung und Gegenüberstellung von erinnerten Lebensabschnitten nicht unbedingt als Möglichkeit, eine traumatische Vergangenheit zu bewältigen, sondern eher als notwendiger Versuch, Ordnung im eigenen Lebensbild $\mathrm{zu}$ schaffen, historische Ereignisse in ihrer Abfolge als daseinsbestimmend zu verstehen, und am allerwichtigsten - zu den Grundquellen einer Identität zu gelangen.

5 Alles, was die deutschsprachige Autorin kroatischer Herkunft behauptet, geht auf die Sprache zurück, die als existentielle Metapher ihr Buch durchzieht. Der Homo- und Heteroreferentialität des sprachlichen Mediums wird ein Denkmal gesetzt, in der „Werkstatt der Wörter" werden neue Artikulationsverfahren geschaffen, das Buch wird zu einer „Huldigung der Sprache“4, einer „Liebeserklärung an die Möglichkeiten der deutschen Sprache" und des linguistischen Zeichens als kommunikative Universalmatrix, einem „spracherotischen Manifest von großer Zärtlichkeit und Überzeugungskraft" ${ }^{\text {“5 }}$.

6 Wenn sie auf zwei linguistisch verschienene Wirklichkeitsebenen Bezug nimmt, sind darunter immer topographische Landschaften zu verstehen, die literarisch verklärt als sprachliche Konstellationen auftauchen. Durch die schriftliche Festigung hybrider Erfahrungen vollzieht sich ein Vorgang der Identitätsaneignung, in dem die zwei verschiedenen kulturellen Identitäten sich $\mathrm{zu}$ einem einheitlichen Gebilde zusammenfügen:

„Das Durchschreiten beider Sprachen kam mir manchmal vor wie zweifaches Leben, wie zwei autonom nebeneinander wirkende Lebensspuren, die zu verbinden mir nur im Schreiben gelang. “6

7 Der Entscheidung für den Erzählakt liegt ein dringendes Bedürfnis zur Identitätstvergewisserung zugrunde: „Mit den Wörtern fängt es an, mich selbst für mich selbst zu geben“". Dieser Entschluss fällt mit dem Wunsch zusammen, „etwas bewahren 
$\mathrm{zu}$ wollen, behüten auch $^{\text {“8 }}$. Dabei wird an die Großvaterfigur erinnert, die entscheidende Kindheitsmomente in Bewegung setzt, um eine rein assoziative, chronologisch ungebundene Bestandsaufnahme und Erkundung eigener Positionierungen im Umgang mit der Sprache in die Wege zu leiten. Das Buch ist über weite Strecken so aufgebaut, dass erzählte Lebenserinnerungen mit reflektiven, abstrahierenden, sentenzartigen Abschnitten abwechseln. Der Erzählfluss weicht oft zurück, bleibt in der Kindheit stehen, dann geht er seinen Weg, nähert sich der Erzählzeit.

8 Das Ich scheint am Anfang, an der Schwelle zwischen zwei Sprachen verfangen zu sein, es ist so gut in der Aufnahmesprache eingebürgert und hat sich dort so gut eingelebt, dass es sich inzwischen von der Herkunftssprache entfernt hat. Als die Hinterfragung weiterschreitet, kommt dieser latenten Sprache, die auch „erste Muttersprache“ genannt wird, eine immer größere Bedeutung zu. Das Slawische wird nur als „Hintergrundmusik ${ }^{\text {“9 }}$ wahrgenommen, diese nimmt an der Sprachmelodie nur begrenzt in ihrer Verdrängtheit teil.

\section{In der Sprache des Aufnahmelandes}

9 Als aber das Ich den Erinnerungsfaden zurückspult, lässt es die zwei Sprachen bei der Ankunft in Deutschland zusammentreffen. Bei der Ausreise entpuppen sich Begriffe wie „Ausland“ und „Gastarbeiter" als erfundene Konstrukte. Ausland wird somit zur Metapher für das versprochene Land auf der „Suche nach einem besseren Leben“, aber auch für die ganze Außenwelt, der man in der Fremde als Unvertrautem begegnet ${ }^{10}$. Das aus einer existentiellen Angst stammende Gefühl einer willkürlichen Ordnung der Signifikanten bringt das Ich zur Feststellung, die Welt ist tatsächlich eine Vorstellung, die ihre Signifikaten als Spielbälle in unterschiedlichen Rastern wirken lässt. In einer Wirklichkeit zweiten Ranges, wo man über menschliche Erfahrungen nachdenkt, kann Fremdheit ihr Recht insofern beanspruchen, als Menschen sich selbst fremd bleiben und an einem wachsenden Entfremdungsvorgang teilnehmen, sich bewusst voneinander entfernend.

Dieser Doppelerfahrung von Angst und Erwartung entspringt eine sich traumatisch vollzogene Anpassung an die neue deutsche Kultur, die Bodrožić im ähnlichen Wortklang „Wunde“ und „Wunder“ auffällt. Die Ankunft bedeutet ein Grenzüberschreiten, ein geographischer Ortswechsel, aber vordergründig ein Sprung „über den eigenen Schatten “11. Das Angstgefühl hat man erst hinter sich, als man schon hinübergesprungen ist. Dabei hat man aber eine lokalgesteuerte, nicht auch die angeborene Identität aufgegeben und in eine neue Haut schlüpfen müssen. Das Neuland wird ursprünglich zwar als „fremd“ empfunden, solange, bis die Verbindung mit den Geschwistern wiederhergestellt wird, bis das Ich sich ihres Dabeiseins vergewissert. Das Deutsche darf insofern „diese Sprache meiner Freiheit" ${ }^{\text {"12 }}$ sein, darf ihr jedoch ohne die sicherheitsspendende Familie immerhin seelisch unvertraut bleiben. Die deutsche Sprache als "Schutzdamm“"13 erfüllt eine Ablenkungsaufgabe und zugleich eine gewissermaßen soteriologische Funktion, da es als eine rein körperliche Rettung stattfindet, die Flucht aus einem kriegsbedrohten Land prägt aber dem Heimatlosen das Flüchtlingsstigma ohne das geistige Einleben in dem Aufnahmeland für immer auf. „Es half mir, das Schreckliche $\mathrm{zu}$ verorten, es aus mir selbst $\mathrm{zu}$ verlagern “14. Die vergangenheitsorientierte Identitätsdimension eines aus einem Kriegsland 
stammenden Mädchens wird somit durch die Berührung mit dem Bild des in Deutschland Beschützten verdrängt. Während die deutsche Sprache als Schutzwall fungiert, ruft die erste Muttersprache schmerzhafte Erinnerungen an das Geburtsland wach. Sprache ist folglich „weder adamitisch noch babylonisch, weder wesenhafter Urlaut noch traurige Konfusion der arbiträren Zeichen: sondern eine Art Los, das man gezogen hat und das die Anweisung auf einen unberechenbaren Gewinn enthält ${ }^{\text {“15. }}$.

11 Als das Ich einsieht, dass Überleben in dem neuen Land allein durch die Aufrechterhaltung von alten Familienbindungen an das Geburtsland möglich ist, wird der Erinnerungsfaden anfangs schüchtern, dann mit immer sicheren Griffen abgespult. Die politische Auflösung Jugoslawiens wird mit der zwangshaften Verstümmelung der Kindheitserinnerungen und dem Verzicht auf die eigene nationalgefärbte Vergangenheit gekoppelt. Die Zerstörung eines Landes im Krieg verläuft parallel zu einem höhnischen Identitätsraub, die neue Zeit verschlingt Vergangenes, betäubt es, lässt es in Ohnmacht fallen: „die Zeit begann Schnaps zu trinken, die Zeit, diese Trinkerin und Betrügerin der Menschen. ${ }^{\text {“16 }}$

12 Über die eigene Ankunft in Deutschland wird nicht ausführlich berichtet, wichtiger scheint der Erzählerin der Augenblick, als Landsleute in Sulzbach in der Nähe von Frankfurt eintreffen. Die Unterscheidung zwischen den zwei Ländern erfolgt in einer chronologischen Anordnung, sie werden aber nicht scharf voneinander getrennt, sondern die Erzählerin bekennt dadurch ihre Zugehörigkeit zu den beiden: „Jetzt kamen schon Leute von unserem ersten Dorf in unser zweites Dorf ${ }^{{ }^{\prime 17}}$. Somit werden zwei Orte einer einzigen Sammelidentität zugeordnet, was dazu berechtigt, sie als gleichwertige Heimatorte anzusehen. Zugleich ist bei der Beobachtung dieses Umzugs der fremde Blick am Werk, der als Doppelsicht - des jugoslawischen Ankömmlings und des jugoslawischen Deutschland-Bewohners - wiedergegeben wird. Beide nehmen in Kategorien der Fremdwahrnehmung aufeinander Bezug, obwohl sie sich ihres gemeinsamen Heimatbedürfnisses bewusst sind: sie kamen „von jenem anderen Hier in dieses andere Hier" ${ }^{“ 18}$. Andersartigkeit trennt die zwei Räume, aber der dringende Anpassungsdrang mahnt, schnell das Herkunftsland in dem Aufnahmeland zu finden, zu schaffen, zu erfinden.

\section{Der Krieg im fernen Jugoslawien}

Selbst das neue Haus in Deutschland, wo die jugoslawische Familie zur Miete wohnt, trägt die Narben eines Krieges, der mit dem sich im fernen Jugoslawien abspielenden parallelisiert wird. Um das Haus schätzen zu lassen, nahm der Vermieter ein einige nach dem Zweiten Weltkrieg auf die Fassade gelegte weiße Platten ab, die er dann nicht mehr anbringen wollte. In der Erinnerung des Kindes überträgt sich der jugoslawische Krieg auf das Wohnhaus, es sind aber nicht vordergründig physiche Schäden, mehr schmerzen die seelischen Leiden. „Er ließ dem Haus dieses Brandmal, und jeder, der an ihm vorbeiging, dachte, es ist das Haus von Ausländern, deshalb sieht es so aus. " ${ }^{19}$ Dass sie dadurch einem natürlichen menschenrechtlichen Status enteignet wurden, dass ihnen die Zugehörigkeit zum neuen Land verweigert wurde, so wird ein Desillusionierungsprozess in Gang gesetzt. Der Übergang von der alten zur neuen Heimat lässt sich schwierig vollziehen, eine neue Identität muss zuerst erworben werden. Das kleine Mädchen denkt an die plattenlosen Hausstellen wie an „entzauberte 
Hauswangen "20, das vorgestellte Zuhause stimmt nicht mit der deutschen Wirklichkeit überein, in der Tat können die jugoslawischen Flüchtlinge ihr Gesicht nicht wahren.

Es treffen dann immer mehrere Menschen aus Jugoslawien ein, im Fernsehen wird über den Krieg berichtet, eine verdrängte Wirklichkeit tritt wieder zum Vorschein. Identität wird im Spannungsfeld der politischen Verhältnisse nicht mehr mit dem Nationalcharakter gleichgestellt, hingegen entpuppt sie sich auf dem Schauplatz einer zunehmenden Verunsicherung als arbiträre Zuweisung, die Menschen zum Kampf gegeneinander treibt. Man flüchtet nach Deutschland nicht aus Feigheit, sondern weil „am Ende das eigene Leben mehr bedeutet [...] als die kroatische oder serbische Identität, die sie bald an irgendeiner Front hätte zum Krüppel machen können." ${ }^{21}$ Nationalgebundene Zuschreibungen weisen kein Individuum als Menschen aus, insbesondere wenn ein Staat infolge politischer Machtverhältnisse und Verhandlungen leicht zugrundegehen kann. Damit scheint auch der Verlust nationaler Identität vorprogrammiert zu sein:

„Ich wollte nichts mehr mit Jugoslawien, nichts mehr mit Kroatien zu tun haben.

Ein eigener Mensch sein, dachte ich jahrelang, das müsste lobenswerter sein als die Identitätskarte eines Landes, das mit einem Mal - und es ist jedes Mal mit einem Mal, mögen die Journalisten und Politiker das heute anders sehen - auseinanderfällt. “"

Man weigert sich, weiterhin seine Identität zu politischen Zwecken instrumentalisieren $\mathrm{zu}$ lassen, vor dem Hintergrund des Krieges erweisen sich starre Identitätsaussagen als lächerlich. In dem kriegsüberlebenden Land mit Beinamputierten und Leuten auf Krücken kann man Worte wie „Kroate, Granate, Granatapfel, Apfelsinne, Cinématograph“23 im gleichen Satz nebeneinander aufzählen oder gegeneinander austauschen. Über die willkürliche Ordnung der Dinge macht man sich in einem sarkastischen Wortspiel lustig, Mord und Zerstörung leben im Alltag mit Nahrungsmitteln und Unterhaltung zusammen. Aber zugleich lassen sich staatliche Zuschreibungen außer Kraft setzen, als das Kind räsoniert, Jugoslawien hätte jedwelchen anderen Namen tragen können, Menschen und Verwandte bildeten das Bindeglied zum Heimatbegriff. Vertrautheit und seelische Nähe gewährten den Zugang zu einer festen Individualität: „Dort kannte man uns, dort waren wir jemand mit einem Namen“24. Es ist „der antrainierte nationale, geographische Reflex“, er

„macht uns glauben, wir bräuchten eine nationale Identität. Warum sagen wir nicht, wir brauchen eine Orientierung und wir möchten die Identitätskarte nennen? So wüßten unsere Kinder, daß die Orientierung nichts Festes ist, daß sie nichts ist, was wir verteidigen müssen, wofür wir sterben und töten wollen." ${ }^{25}$

Unter den Umständen einer ständigen Gefährdung des Autochtonen durch die politischen Umwälzungen bringt die Behauptung von Nationalgefühlen keine Rettung des nationalistisch geprägten Selbstbildes mit sich. Die Folgen vielseitiger kultureller Durchdringungsprozesse in den immer breiter aufgefächerten Weltzusammenhängen zeigen sich bei am heute viel beschworenen Globalisierungsphänomen, was die Autorin zum Schluss kommen lässt, das Verbindende zwischen den Menschen und Völkern müsse in der Bewahrung des „Ureigenen“ ${ }^{26}$ gesucht werden. Wenn sich die Erzählerin zum Beispiel an ihre Heimat erinnert, muss sie schwermütig auf das Wort „alma“ in der Ortbezeichnung „Dalmatien“ stoßen. „Seele“ und „Herz“ sind diesem Gebiet die Kosenamen, die Eltern benutzen, wenn sie ein Kind rufen. Darum empfindet sie diese Tätigkeit als „wesenhaften Ruf “27 zu den Quellen menschlichen Daseins, zur Liebe, die sich in jede Sprache der Welt übertragen lässt. Ohne die Verbindung mit dem Herkunftsland wiederherzustellen, kann sich der Ankömmling in einem anderen Land 
eine neue Identität durch den Übergang von der alten nicht verschaffen. Das kleine Kind ist von den sauberen deutschen Ortsschildern und die Welt, die es kennenlernt, beeindruckt, aber erkennt zugleich, dass sie sich „niemals über die Liebe legten“ und muss sich an die kleine Dorfschule zuhause als identitätsstiftenden Maßstab erinnern ${ }^{28}$.

\section{Grenzen und Identitäten}

Was eigentlich das ganze Buch leitmotivisch wie einen roten Faden durchzieht ist der Gedanke, dass die politische Zerspaltung des Herkunftslandes keine Schaden in der Heimat-Wahrnehmung zugefügt hat. Grenzen innerhalb des ehemaligen Jugoslawien erweisen sich als künstlich, Zugehörige desgleichen Raums erkennen sich wieder, reichen sich die Hände. „Der Krieg wird unsere Liebe niemals zerstören, niemals vollständig auslöschen können“29, ruft die ursprüngliche Identität aus. Zur Veranschaulichung werden drei Begegnungen geschildert, die in verschiedenen europäischen Städten stattfinden, von Rom über Paris in Frankfurt. Die Erzählerin befindet sich schon seit dem neunten Lebensjahr in Deutschland und ist inzwischen eine Erwachsene geworden. Immer noch begeistert von der Fähigkeit der deutschen Sprache, Klarheit in ihren Lebensvorstellungen zu schaffen, hatte sie am Anfang bereits behauptet, nur diese Sprache verhelfe ihr, ihre Identität verorten zu können. Und trotzdem stellt sich heraus, dass in der Fremde die alten Wurzeln zum Vorschein treten. Als sie in einem Bus in Rom von einer Frau auf Englisch angesprochen wird, fällt ihr die jugoslawische Aussprache auf, über die Wörter stellt sich eine alte schicksalshafte Verbindung her, die bis in die tiefe Vergangenheit reicht und tiefe mystische Gebetsklänge aufnimmt. Fremdheit wird somit stark in Frage gestellt, die Entdeckung eines vertrauten gemeinsamen Erfahrungshorizontes verbindet Dazugehörige über das Mittel der Sprache:

„Die Wörter, wie Vebündete, reichten sich zwischen uns und unseren Brustkörben die Hände, während wir, zwei eigentlich einander fremde Menschen, unsere Hände hielten und dabei immer hin- uund hertänzelten, als seien wir schon zusammen in den Kindergarten gegangen. “30

18 In Paris ist es wieder das sprachliche Medium, das Nähe schafft. Ein Plakat mit der Ankündigung eines Bregović-Konzertes führt sekundenschnell das Heimatbild vor Augen und übermittelt eine ferne Botschaft. Beim Erlernen des Französischen stellt die Erzählerin überrascht die Latenz des Serbokroatischen fest, einer Sprache, die auf der Kontaktebene mit dem Anderen als identitärer Bestandteil wieder zum Leben erweckt wird. Auch in Paris erblickt an einer Haltestelle einen Mann, an dem sie „etwas Jugoslawisches" sieht. Als ihm seine Zeitung mit kyrillischer Schrift aus der Tasche rutscht, bewahrheiten sich die Vermutungen. Wieder sprechen sie „aufgeregt wie Kinder miteinander ${ }^{\text {“31 }}$, in der Einsamkeit des neuen Wohnorts bleibt sie in den alten Erinnerungen versunken: „er wohnte am Ende der rue Oberkampf. Zusammen mit seiner Frau. Ich ungefähr in der Mitte der langen Straße. Zusammen mit meiner jugoslawischen Vergangenheit." ${ }^{\text {"32 }}$

Auf der Reise nach Paris wird sie wieder von einer jugoslawischen Landsfrau angeredet, am Frankfurter Bahnhof steigt eine Frau mit Blumenkopftuch mit ihr in den Zug ein, segnet sie in der bekannten Herkunftssprache und entfernt sich dann. Es findet dabei wieder ein Sakralakt statt, eine erneute Einweihung in die geheimnisvollen Tiefen einer inzwischen vergessenen Daseinsebene. Der in der weiten Welt herumirrende 
Erwachsene kehrt zu den Quellen der eigenen Kindheit und Individualität zurück. Die

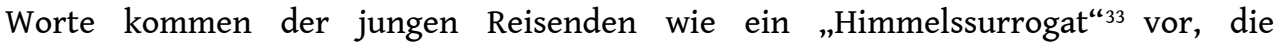
Blumenkopftuchfrau nimmt sie wie ein Engel in Schutz ${ }^{34}$.

Das Nachdenken über die Versprachlichung identitärer Erfahrungen löst einen inneren Kampf mit der eigenen Vergangenheit aus, die bewältigt und als solche angenommen werden muss. Die Erzählerin bekennt ihre Ahasver-Lage, Sprechen wird zum Handeln, als ihr an den Wörtern ihrer Landsleute, denen sie an verschiedenen Orten Europas hinterhergeht, ihr eigenes Verfangensein an Nahtstellen zwischen den Kulturen bewusst wird. Wörter sind wie Menschen ,weitgereiste Vögel“ ${ }^{\text {“35 }}$, eine feste und einzige Identität muss nicht aufgezwungen werden, da Grenzen selbst erfundene Gebilde sind, die einen zur Rechenschaft ziehen:

„Und auch begriff ich, wie absurd es eigentlich ist, ein Paßbesitzer zu sein, etwas so Äußeres sein zu müssen uns es zu werden, weil man hier auf dieser Erde ein Jemand ist, wenn man sich an irgendeiner ausgedachten Grenze als Einheit von Gesicht und Name ausweisen kann. “36

Zum Beispiel wird sie während des Pariser Aufenthalts für eine Argentinierin gehalten, was sie zugleich wundert und ironisch folgern lässt, nun habe sie endlich eine klare, „ganz leicht erkennbare ${ }^{{ }^{37}}$ Identität. Bei einem Konzert in der kroatischen Hauptstadt stellt die aus dem Ausland Zurückgekehrte fest, dass Musik insofern verbindend wirkt, als Bürger des ehemaligen Jugoslawien sich als Gemeinschaft mit einer einzigen Stimme behaupten. Über politisch verordnete Zugehörigkeit hinaus kommt das

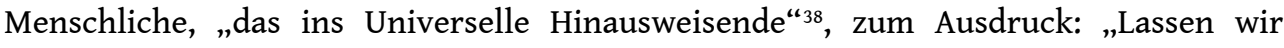
Fahnen Fahnen sein. Seien wir Menschen. “39

Nach der erfolgreichen kathartischen Beichte, wo in der deutschen Sprache die Vergangenheitsbewältigung angestrebt wird, wendet sich Bodrozic von erinnerten biographischen Abschnitten zur Konzeptualisierung von Erlebnissen im Umgang mit Wörtern. Die Sprache, in der bisher Berichte erstattet wurden, verwandelt sich in eine Metasprache, in der über das Verhältnis zu Gesagtem und Ungesagtem nachgedacht wird. Der Erzählton wird lockerer, die Autorin staunt über die Entdeckung von Wortlandschaften und -offenbarungen, sie setzt Wörter aus verschiedensten Bereichen in Verbindung zueinander und hinterfragt ihre verborgenen Bedeutungen. Der Schriftsinn ist ihr „ein vielfacher, schichtenweise abgetragen und als offenbarung neu zusammengesetzt“, Marica Bodrožić „liest das Deutsche wie die alten Theologen die Heilige Schrift. “"

In der zweiten Buchhälfte kommt die Dichterin zum Vorschein, ihre Stimme setzt sich ausdrucksstärker mit empfindsamen Beobachtungen und poetischen Wortschöpfungen durch. Ein Beispiel in diesem Sinne ist das Leitmotiv des Sterns (näher anzuschauen), das dem Buch seinen Titel gibt. Leitmotivische Einschübe treten hauptsächlich an Nahtstellen auf, werfen Fragen auf und begleiten den Weg, den die Autorin von der Thematisierung der Fremdheitserfahrungen zur Erkenntnis einer weltübergreifenden, versöhnenden Menschlichkeit macht. Nicht anders als kosmisch kann das menschliche Dasein aufgefasst werden. Jeder Einzelne ist „ein Bewohner jenes großen kosmischen Hutes“, wo auch Sprachen als „faßbare Gestirne“ anzutreffen sind ${ }^{41}$. Wörter sind „eine Sternensaat des Hierseins. Eine Ernte. Ein Segen“" ${ }^{\text {“2 }}$ Jede Kindheit „trägt das Erbe der Sterne in sich “43, Körper brechen zusammen, wenn Sterne „keinen Zugang zu unserem Brustland haben " ${ }^{44}$. Beim Anblick des Sternenhimmels wird man in die Geheimnisse der eigenen Herkunft eingeführt. Das Wortspiel zwischen „erben“ und „färben“ deutet auf 
das Verhältnis zwischen „emotional assimilierter“ Vergangenheit und eigenständiger Fortsetzung in der Zukunft hin: „die Sterne als Lichtsymbole kennzeichnen die Kontinuität des Erbes“, das von jedem Einzelnen „verwertet“ und „mit den eigenen Farben versehen" werden muss ${ }^{45}$.

„Wie stünde es um uns, wenn wir das Erbe der Sterne in uns verlebendigen könnten und es ein Sternbuch gäbe, in allen unseren Fingerkuppen abrufbar wäre, und wir die Kuppen wie Bücher lesen müßten, um zu überleben. Wie einfach wäre unser Leben, wenn wir die Sterne erben und färben könnten, wie es unserem Glück beliebt! ${ }^{46}$

\section{Primärliteratur}

Marica Bodrožić, Sterne erben, Sterne färben. Meine Ankunft in Wörtern, Frankfurt am Main, Suhrkamp, 2007.

\section{Sekundärliteratur}

Alexander Czajka: Marica Bodrožić: Sterne erben, Sterne färben. Meine Ankunft in Wörtern. Buchbesprechung vom 03.12.2007, http://www.buecher.de/suche/marica-bodrozic.

Burkhard Müller: "Marica Bodrožić. Sterne erben, Sterne färben. Meine Ankunft in Wörtern". In: Süddeutsche Zeitung, 13. Juni 2007.

Dagmar Winkler: Marica Bodrožić schreibt an die „Herzmitte der gelben aller Farben“. In: Michaela Bürger-Koftis (Hg.): Eine Sprache - viele Horizonte... Die Osterweiterung der deutschsprachigen Literatur. Porträts einer neuen europäischen Generation. Wien: Praesens 2008, S. 107-119.

Klaus Hübner: "Der Plural ist mein tägliches Brot”. Marica Bodrožić - eine deutsche Dichterin aus Dalmatien. In: literaturkritik.de, Nr. 1, Januar 2009.

\section{NOTES}

1. Dieser Artikel erscheint mit der finanziellen Unterstützung des EU-Projekts « Die Entwicklung der Innovationsfähigkeit und die Erhöhung der Forschungsauswirkung durch PostdocProgramme ", gehörend zum operativen sektoriellen Programm zur Entwicklung des Personals POSDRU (89/1.5/S/49944), Priorität-Achse 1, Einsatzbereich 1.5 « Doktoratskollegs und PostdocForschungsprogramme zur Unterstützung der Forschung». (This article appears with the financial support of the project « Developing innovation capability and increasing the impact of post-doctoral research programs", part of the Sectoral Operational Programme Human Resources Development - POSDRU (89/1.5/S/49944), Priority Axis 1, area of intervention 1.5 «Doctoral and post-doctoral research support»). Alexander Czajka: Marica Bodrožić: Sterne 
erben, Sterne färben. Meine Ankunft in Wörtern. Buchbesprechung vom 03.12.2007, http:// www.buecher.de/suche/marica-bodrozic.

2. Marica Bodrožić, Sterne erben, Sterne färben. Meine Ankunft in Wörtern, Frankfurt am Main, Suhrkamp, 2007, S. 11.

3. Ebd.

4. Walter Hinck: „Flieger, grüß ihr die Sonne. Na also: Marica Bodrožić frischt die deutsche Lyrik auf“. In: Frankfurter Allgemeine Zeitung, 31. August 2007.

5. Klaus Hübner: "Der Plural ist mein tägliches Brot". Marica Bodrožić - eine deutsche Dichterin aus Dalmatien. In: literaturkritik.de, Nr. 1, Januar 2009.

6. Ebd., S. 96.

7. Ebd., S. 67, Herv. im Original.

8. Ebd., S. 12.

9. Ebd., S. 14.

10. Ebd., S. 20.

11. Ebd., S. 22.

12. Ebd., S. 23.

13. Ebd., S. 27.

14. Ebd., S. 29.

15. Burkhard Müller: "Marica Bodrožić. Sterne erben, Sterne färben. Meine Ankunft in Wörtern". In: Süddeutsche Zeitung, 13. Juni 2007.

16. Ebd., S. 25.

17. Ebd., S. 26.

18. Ebd.

19. Ebd., S. 28.

20. Ebd., S. 29, kursiv im Original.

21. Ebd., S. 30.

22. Ebd., S. 37, Herv. im Original.

23. Ebd., S. 39.

24. Ebd., S. 61.

25. Ebd., S. 56.

26. Ebd., S. 46.

27. Ebd., S. 31.

28. Ebd., S. 38.

29. Ebd., S. 43.

30. Ebd., S. 42.

31. Ebd., S. 73.

32. Ebd.

33. Ebd., S. 54

34. „Bog ti pratio svaki tvoj korak. Gott begleite jeden Deiner Schritte. Uvjek ti Bog držao tvoju ruku. Stets halte Gott Deine Hand. Bog ti ljubio svaku tvoju suzu. Gott küsse jede deiner Tränen". Ebd., S. 55.

35. Ebd., S. 66.

36. Ebd., S. 67, Herv. im Original.

37. Ebd., S. 75.

38. Walter Hinck: „Flieger, grüß ihr die Sonne. Na also: Marica Bodrožić frischt die deutsche Lyrik auf". In: Frankfurter Allgemeine Zeitung, 31. August 2007.

39. Ebd., S. 78, Herv. im Original.

40. Burkhard Müller: „Marica Bodrožić. Sterne erben, Sterne färben. Meine Ankunft in Wörtern“. In: Süddeutsche Zeitung, 13. Juni 2007.

41. Ebd., S. 22. 
42. Ebd., S. 67.

43. Ebd., S. 41.

44. Ebd., S. 84.

45. Dagmar Winkler: Marica Bodrožić schreibt an die „Herzmitte der gelben aller Farben“. In: Michaela Bürger-Koftis (Hg.): Eine Sprache - viele Horizonte... Die Osterweiterung der deutschsprachigen Literatur. Porträts einer neuen europäischen Generation. Wien: Praesens 2008, S. 107-119, hier S. 118. 46. Ebd., S. 89.

\section{RÉSUMÉS}

Marica Bodrožićs Prosaband Sterne erben, Sterne färben (2007) ist ein Versuch, durch eine Hin- und Herreise, die sowohl zurück in die Vergangenheit als auch weiter bis in die Gegenwart schreitet, eine seelische Biographie zu erkunden. Die Schriftstellerin kroatischer Herkunft lebt seit dem zehnten Lebensjahr in Deutschland. Dieser empfindungsbeladenen Topographie liegt die Frage nach dem Vorrang einer sprachlich erzeugten Seelenlandschaft zugrunde, in der Wörter verschiedener Sprachen und Dialekte zusammenwohnen, wobei jede nationale Prägung zugleich einen ins Emotionale gesteigerten Einfluss ausübt. Die Verfasserin stellt sich als Fragende und Suchende dar, die zwischen zwei Identitäten pendelt. Jugoslawische Vergangenheit, der Ankunft in dem deutschen Aufnahmeland, das neue hessische Dorf, neuere Erfahrungen der Erwachsenen in europäischen Großstädten, alles kreist um das identitätserforschende Unterfangen, Trennlinien zu entdecken und zugleich Brücken zwischen Menschen und Kulturen zu schlagen.

Le texte en prose de Marica Bodrožić tente d'explorer une biographie de l'âme dans un mouvement de va-et-vient entre le passé et le présent. L'écrivaine d'origine croate vit en Allemagne depuis l'âge de dix ans. Cette topographie émotionnelle conduit à s'interroger sur la prédominance d'un paysage de l'âme produit par la langue dans lequel cohabitent des mots de langues et dialectes différents, chaque caractéristique nationale exerçant une influence qui devient émotion. L'auteure se présente comme instance chercheuse et questionnante, oscillant entre deux identités. Le passé yougoslave, l'arrivée dans le pays d'accueil qu'est l'Allemagne, le nouveau village hessois, les expériences récentes de l'adulte dans plusieurs grandes villes européennes - l'exploration de l'identité se concentre sur la quête des lignes de démarcations et en même temps sur les liens à établir entre les hommes et les cultures.

Marica Bodrožićs prose text Sterne erben, Sterne färben (2007) is an attempt to explore a biography of the soul by means of a to and fro journey between the past and the present. The writer of Croatian origin has lived in Germany since the age of ten. This emotional topography is based on the question of the priority of a linguistically generated soul-landscape, in which words of different languages and dialects cohabit. The author presents herself as a questioner and a seeker who commutes between two identities. The Yugoslavian past, the arrival in the host country, Germany, the new Hessian village, recent experiences of adults in European cities, everything revolves around the quest for an identity and the will to discover differences and build bridges between peoples and cultures. 
INDEX

oeuvrecitee Sterne erben, Sterne färben

\section{AUTEURS}

RALUCA RĂDULESCU

Universität „Al. I. Cuza“ Iaşi (Rumänien) 